\title{
Sabotages et lutte armée
}

Entretien de Georges Delepaut avec Yves Le Maner, 22 juin 2004

\section{Laurent Seillier}

\section{OpenEdition}

\section{Journals}

Édition électronique

URL : https://journals.openedition.org/rhcf/543

DOI : $10.4000 /$ rhcf.543

\section{Éditeur}

Rails \& histoire

\section{Édition imprimée}

Date de publication : 1 juin 2006

Pagination : 151-172

ISSN : 0996-9403

\section{Référence électronique}

Laurent Seillier, "Sabotages et lutte armée », Revue d'histoire des chemins de fer [En ligne], 34 | 2006, mis en ligne le 13 mai 2011, consulté le 22 avril 2022. URL : http://journals.openedition.org/rhcf/543 DOI : https://doi.org/10.4000/rhcf.543 


\title{
Sabotages et lutte armée \\ Entretien de Georges Delepaut avec Yves Le Maner, 22 juin 2004
}

\author{
Laurent Seillier \\ La Coupole
}

Cet entretien a été réalisé, 60 ans après la libération de la région Nord - Pas-de-Calais, à une période charnière de notre histoire, celle où sont en train de disparaittre les derniers témoins et acteurs de la Seconde Guerre mondiale. Aussi la Coupole, Centre d'histoire et de mémoire du Nord - Pas-de-Calais a-t-elle entrepris, à cette occasion, un recensement puis un programme d'enregistrements vidéo numériques de personnes dont l'histoire personnelle pouvait être utile à la compréhension de l'histoire de la Zone interdite, région correspondant aux deux départements du Nord et du Pas-de-Calais, qui ont été rattachés au commandement militaire de Bruxelles par les conditions d'armistice. Parmi ces personnes figure Georges Delepaut, cheminot résistant, dont l'action peut être considérée comme une des formes emblématiques de la Résistance dans les deux départements nordistes, résistance aux caractères particuliers s'expliquant par l'histoire politique, économique et militaire de la région et par sa géographie.

La résistance dans la région Nord - Pas-de-Calais commence dès le mois de juin 1940. Cette précocité peut s'expliquer par une haine féroce des Allemands et par une anglophilie prononcée, souvenirs du premier conflit mondial, le nord de la France ayant été à la fois zone de combats avec une forte présence de troupes britanniques et une des seules régions de France occupée par les armées de Guillaume II. Les premiers actes de résistance (cache de soldats anglais, récupération d'armes...), émanent le plus souvent d'initiatives individuelles et concernent toutes les catégories de population. Durant l'hiver 1940-1941 apparaissent les premiers journaux clandestins appelant à la Résistance et au patriotisme. L'été 1941 est un tournant : l'entrée en guerre de l'Allemagne nazie contre l'URSS déclenche, dans le bassin minier, de véritables actions de guérilla des communistes contre l'occupant. L'année 1942 voit la naissance et la structuration de grands réseaux, la plupart sous l'égide de Londres, le MI6 pour le renseignement et le SOE (Special Operation Executive) pour le sabotage. Mais ce n'est véritablement qu'à partir de 1943 que les réseaux action sont opérationnels, à l'exemple de l'équipée 
du réseau «Farmer» menée à Lille par les hommes du capitaine Trotobas auxquels s'est joint G. Delepaut, engagé dans les FTP, pour le sabotage du dépôt de Tourcoing qu'il raconte. Mais cette année 1943 est aussi particulièrement difficile, puisqu'elle voit le démantèlement de grands mouvements tels l'OCM (Organisation civile et militaire) et la Voix du Nord par les forces de répression nazies. Des dizaines d'arrestations sont effectuées; ceux qui y ont échappé doivent entrer dans la clandestinité pour plusieurs mois. À la veille du 6 juin, la résistance régionale apparaît donc particulièrement épuisée. Elle parvient, cependant, à mettre en œuvre aux lendemains du débarquement le Plan vert (sabotages ferroviaires), le Plan violet (coupures de lignes téléphoniques) et le Plan tortue (ralentissement des mouvements des unités allemandes). Début septembre 1944, la région est libérée par les armées alliées aidées des FFI.

Georges Delepaut fut démobilisé à la fin d'octobre 1945 et a repris alors son métier de cheminot au dépôt de Tourcoing. Un énorme travail de remise en état du chemin de fer l'attendait.

Cette transcription a été réalisée en mai 2006 à partir du film par Yolande Sanguine (AHICF). L'usage de la virgule, non grammatical, tente de restituer la scansion du discours. Le film peut être visionné à La Coupole (BP 284, 62504 Saint-Omer cedex, tél. : + 33 (0)3 2112 2727, fax: + 33 (0)3 21392145 , http:/ / mm:lacoupole.com/) et à l'AHICF, pour une consultation individuelle entrant dans un cadre scientifique. 


\section{Entretien de Georges Delepaut avec Yves Le Maner, 22 juin 2004}

- Alors Georges, sill te plait, on va faire les présentations...

- Oui.

- Peux-tu, s'il te plait, nous dire où et quand tu es né?

- Alors, je suis né à Tourcoing le 9 mai 1923, et dans une famille, dont la mère a subi l'occupation pendant les quatre ans, 14-18, avec déjà toutes les exactions qui se sont produites à l'époque, et mon père, lui, mobilisé, grand mutilé de la guerre 14-18.

- Donc ily a un héritage très fort, dans la famille, de la Première Guerre mondiale?

- Exactement.

- Qu'est-ce que ta mère t'a raconté, quand tu étais enfant, de ce qu'elle a connu pendant les quatre années?

- Eh bien, elle m'a raconté tout ce qui c'était passé, les restrictions, les interdictions, les hommes qui étaient réquisitionnés pour le travail les brassards rouges à l'époque - ce qui fait que chez moi, je n'ai jamais entendu le mot «allemand ». On ne parlait que des «boches», comme beaucoup de ma génération de, du secteur. J'ai été élevé dans la haine du boche, et..., j’ai l'impression que ça doit expliquer, peut-être, un petit peu, le fait que dans notre région, la résistance se soit développée, un peu plus que dans d'autres zones de France.

- Est-ce que ton père te parlait de sa vie dans les tranchées?

- Il m’en parlait de la guerre, mais il a été blessé assez rapidement, en 1915, il a été blessé gravement, puisqu'il était pensionné dès le départ à $75 \%$ et porté en aggravation à 100 par la suite, et il a été hospitalisé pratiquement, dès 1915, et il était à Limoges, mais il m’a évidemment parlé de toute cette période, qu'il a connue dans la Marne, où là, tous les combats qui se sont produits là, eh bien, il a vu de nombreux camarades à lui tomber, hein, et surtout blessés également, hein, parce que l'hécatombe a été très importante. Alors ça, je l'ai entendu raconter de très nombreuses fois, et il fallait un peu le pousser pour qu'il en parle, mais il me l'a raconté quand même.

- La Coupole / Georges Delepaut. Citation autorisée dans un cadre scientifique à 
- Donc, en fait, tu es né dans une famille où ily a une culture de guerre?

- Il y a une culture, de guerre et, je crois, d'hostilité aux Allemands, à l'Allemagne. Ce qui fait qu'en 39, quand la guerre s'est déclarée, eh bien pour nous, c'était déjà, pour nous c'était l'ennemi.

- Alors là, arrive donc un choc considérable pour la France et le nord de la France en particulier, c'est linvasion du mois de mai 1940. Peux-tu nous dire comment les événements se sont déroulés pour toi et ta famille?

- Alors là, évidemment, le souvenir de 14-18 avec bien sûr, tout ce qui s'y était passé, a incité notre famille, enfin mon père, des voisins, des cousins, à partir, surtout les hommes, même des familles entières sont parties, par contre, ma mère, $j$ 'avais des sœurs en bas âge, puisque la dernière avait six ans, par conséquent, elle est restée à la maison, mais par contre nous, nous sommes partis. Et nous avons été bloqués à Hesdin. À Hesdin où il y avait eu des parachutistes qui avaient livré combat, et je me souviens que nous sommes restés allongés pendant $24 \mathrm{~h}$ dans un fossé, sans pouvoir relever la tête tellement ça y allait, et puis le lendemain matin, mon père et un cousin plus âgé avaient réussi, eux, au début de cette fusillade à passer le long du parapet du pont au-dessus de la Canche, par contre, les trois jeunes, non expérimentés, on était resté bloqués et nous nous sommes retrouvés le lendemain matin sur ce pont, sur la Canche, qui n'est pas très large, au milieu des cadavres, il y en avait au moins cinquante autour de nous.

- Donc, en fait, tu vois la réalité de la guerre.

- On voit la réalité de la guerre. Évidement, bloqués, ben, on décide de rentrer, mais retournant, pour rentrer vers Saint-Pol, les Allemands ne nous ont pas laissé passer. Ils nous ont déviés par la route vers Anvin, parce que Saint-Pol brûlait. Et pendant 15 ou $20 \mathrm{~km}$, nous étions à vélo, et là, on nous a obligés à rouler sur la gauche. Et toute une colonne motorisée allemande circulait sur la droite, s'arrêtait, repartait, ainsi de suite, et on a fait des aller-retour en fait. Ils nous doublaient, on les redoublait, et puis ils ont disparu et on s'est retrouvé dans la population française, dans des unités françaises, il y avait des soldats français, des officiers. On s'est retrouvé également parmi les Anglais, et évidemment, toute cette colonne de motorisés qui avait dû passer par Sedan, pour rejoindre la mer, explique évidemment que tout ça c'était emprisonné, et on est allé jusqu'à Vieux-Berquin où là un cousin connaissait des personnes, on est resté un ou deux jours. 


\section{- Comment tu percevais l'état d'esprit des gens que tu croisais?}

- Eh bien là, les gens, alors, je me souviens qu'on avait croisé un brave prêtre assez âgé, avec des vieilles personnes, dans une carriole attelée à un cheval qui partait pour évacuer, lui aussi. Et on lui dit : « Ne partez pas, c'est pas la peine, on a vu des Allemands, ils sont déjà làbas, nous on a été bloqués, on retourne. » On a expliqué, mon père a même expliqué ça à un officier français qui l'accusait d'être un espion, et on a expliqué : « Non, écoutez, on a vu ça », et à VieuxBerquin on s'est retrouvés pendant quelques jours, et on est rentrés à Tourcoing, mais alors là, pas moyen de regagner notre domicile qui se trouvait très près de la frontière belge. Les Anglais qui étaient toujours là, avaient fait un barrage à quelques centaines de mètres de la frontière, ce qui fait que nous, mon père et moi, nous ne pouvions pas regagner notre domicile. Et il a fallu attendre quelques jours et, un matin, les Anglais plus là, et on a pu regagner. Et est arrivé début juin, bien sûr, et je me souviens de la période du 16-17 juin, le 17 juin en particulier. J'ai entendu, comme beaucoup de voisins, le maréchal Pétain qui a annoncé, dans un message qui a été diffusé par la radiodiffusion française toute la journée, le message dans lequel il annonce qu'il s'est adressé à l'adversaire pour demander les conditions d'armistice. Par contre, le lendemain, le 18, alors là..., comme la plupart des gens, personne n'a entendu l'appel du général de Gaulle. Mais, un autre point, c'est que toutes les personnes que j'ai connues et qui ont entendu Pétain annoncer qu'il avait demandé les conditions d'armistice, je ne me souviens pas d'avoir entendu une seule personne critiquer Pétain d'avoir demandé les conditions d'armistice. Au contraire et tout le monde disait : « Bravo Pétain, il a arrêté la guerre, il n'y aura plus de tués. » C'était la réaction...

- À chand!

- Ah oui, tout le monde, tout le monde.

- Mais enfin, très vite, ily a une autre donnée qui entre en jeu, c'est que ily avait les Allemands surplace.

- Il y a les Allemands sur place avec évidemment,

- et ces Allemands... [inaudible]

- Après, le souvenir... dans la vie quotidienne, au début, ils semblaient vouloir être très doux, il n'y avait pas d'exaction, y avait rien qui se passait, par contre, évidemment, il y avait des affiches annonçant qu'il fallait absolument que les militaires qui n'avaient pas été 
prisonniers, qui étaient rentrés chez eux, devaient se présenter à la mairie, bon, ben, ce qui fait qu'un mes oncles qui était tailleur et service auxiliaire, qui avait... est allé et bien voilà, et il a été fait prisonnier. Et, bien sûr, au bout d'un certain temps, c'étaient les postes qu'il ne fallait plus écouter, surtout Londres bien sûr, des postes qui ont été réquisitionnés, et ramassés parce que bon...

\section{- À l'époque tu es cheminot?}

- Je suis cheminot, je suis à l'école, aux ateliers d'Hellemmes, à l'école de formation des techniciens pour l'entretien du matériel ferroviaire, en particulier, le matériel roulant, c'est-à-dire matériel moteur et matériel remorqué.

- Alors, quand on est un jeune cheminot - la guerre tu vas la passer entre 17 et 21 ans - quelle est ta vie quotidienne? Imagine une journée ordinaire, à quelle heure te lèves-tu, qu'est-ce qui se passe dans ta journée?

- Alors, immédiatement après l'armistice demandé par Pétain, le..., toutes les industries étaient au repos, plus personne ne travaillait, nous non plus aux chemins de fer, et puis en juillet, vers mi-juillet à peu près, j'ai reçu un courrier, disant qu'il fallait reprendre, j'ai donc repris la route des ateliers d'Hellemmes et continué ma formation. Et dès 1942, mon père qui commençait à avoir par un de ses camarades qui était Front national, des journaux, des tracts, eh bien, j'en prenais, et puis je les transportais à Hellemmes où je faisais une diffusion. J'ai commencé en fait un peu comme ça. Mon père en fait m'a entraîné un peu.

- Avant qu'on en vienne à ton action résistante, peux-tu nous décrire une journée ordinaire d'un jeune sous l'occupation dans le nord de la France?

- Eh bien, au début les transports en commun n'étaient pas encore rétablis, en particulier les trains, et je me souviens qu'on faisait le trajet de Tourcoing à Hellemmes, le matin et le soir au retour, en bicyclette. Et, bien sûr, il fallait partir assez tôt puis on rentrait assez tard, puisque les journées de travail étaient beaucoup plus longues à ce moment-là qu'actuellement, hein. Et bien sûr on mangeait au restaurant d'entreprise, mais assez rapidement évidemment les restrictions ont commencé à apparaître, et puis disons que le ravitaillement était un petit peu plus spartiate et il fallait essayer de trouver. Le rationnement a commencé assez rapidement, et, à partir de là il a fallu commencer, en dehors des journées de travail des uns et des autres, à essayer de partir par le train, à droite, à gauche, dans les fermes, dans les Flandres en particulier, pour essayer d'avoir un 
peu de ravitaillement supplémentaire, des pommes de terre, des haricots, et malheureusement, lorsque l'on rentrait par le train, on repassait obligatoirement par Lille, il est arrivé à plusieurs reprises qu'une saisie générale de tout ce qui descendait du train était faite par la police française. De mes souvenirs, je ne me souviens pas que les Allemands aient, euh...

- Ça ne les intéressait pas...

- ... pris tout ça. C'était la police française, ce que ça devenait je n'en sais rien, mais enfin à plusieurs reprises c'est arrivé. Tout ce qui descendait, et hop, tout était pris, entassé.

- Alors, là, on va en venir maintenant à ton action résistante. On n'est pas dans les films hollywoodiens, c'est-à-dire ça se fait petit à petit, par des tas de petites touches d'abord individuelles, et puis des petits groupes. Comment tu es venu à être impliqué dans des actions résistantes?

- Bon, alors, ateliers d'Hellemmes, dès que mon père avait donc des journaux j'ai commencé à faire un peu de diffusion de journaux et tracts du Front national, les seuls qu'on avait à l'époque et...

- Est-ce que tu peux préciser pour les jeunes d'aujourd'bui que le Front national de l'époque était...

- Ah, le Front national, évidemment, celui de l'époque, le Front national de libération de la France, hein, qui n'avait rien à voir avec l'organisation politique qui a pris le même nom maintenant, et c'était un mouvement qui avait été créé à l'initiative du Parti communiste d'abord, d'ailleurs, mais j'y ai trouvé, moi, des gens de toutes tendances et même j'y ai connu un prêtre. Ce qui veut dire que dans ce mouvement il y a eu quand même des gens de tous les bords. Bien qu'une majorité de communistes s'y trouvait, c'est vrai, mais il y avait des gens de tous les bords. Et, euh... je finis ma formation à Hellemmes en 1942, et le $1^{\text {er }}$ septembre 1942 , je suis nommé au dépôt de locomotives de Tourcoing, un des techniciens chargé de l'entretien du matériel moteur par conséquent. Alors là, évidemment personne ne me connaît, mais bien sûr, 1942, il y avait déjà des revers en Russie, il y avait des événements en Afrique du Nord qui se passaient, les avances et reculs, avec Rommel et puis les Anglais de l'autre côté, eh bien, évidemment, dans la journée, on était amené, puisque tout le monde écoutait plus ou moins la radio anglaise bien entendu, on était amené à discuter de ces événements, de ce qui se passait, d'un côté et de l'autre. Et, bien sûr, ceux qui étaient déjà 
engagés dans un mouvement repéraient ainsi le terrain favorable pour être contacté, ou celui qu'il fallait éviter. J'ai donc été assez facilement repéré comme terrain favorable.

- Et ton père en plus?

- Ah non, mon père n'était pas cheminot, il travaillait dans une...

- Il avait des connexions déjà avec le Front national?

- Oui, oui, oui, avant moi, dès 1942, puisque le 2 mai 1942, j’avais commencé à diffuser des journaux. Mais là, c'est le Front national qui m'a contacté, puisque à l'époque, c'est donc en septembre 1942, par la suite c'est le réseau Sylvestre qui a été très implanté dans le milieu cheminot, fin 42 , début 43 en particulier. Donc à partir... Si ça c'était produit plus tard, peut-être aurais-je été contacté tout simplement par le réseau Sylvestre et puis je serais entré dans ce réseau-là. On entrait dans un réseau par le contact qu'on avait, qu'on ne choisissait pas.

- Professionnel...?

Alors, pour essayer de bien poser le décor, dans un dépôt comme celui de Tourcoing, ily a là-dedans des cheminots allemands, des cheminots de la Reichsbahn, quels sont les rapports entre les cheminots allemands et les cheminots français?

- D’abord, il faut savoir que les cheminots allemands qui étaient présents dans toutes les emprises SNCF, partout, même aux ateliers d'Hellemmes il y en avait, étaient en uniforme et étaient armés. Ils étaient donc considérés comme des militaires. Aucun d'eux ne parlait le français, il y avait donc des interprètes et disons que les contacts se faisaient essentiellement, tout au moins pour nous le personnel, par des interprètes qui nous parlaient de ceci ou de cela, et c'est tout. Par contre, au niveau direction, il y avait des contacts plus rapprochés bien entendu, par l'intermédiaire des interprètes également.

- D'accord. Donc, là, on est dans cet atelier, au dépôt de Tourcoing et, petit à petit, tu prends des responsabilités, des engagements plus forts?

- Oui, alors, le contact avec le Front national a été un contact Front national pur au départ. C'est-à-dire que j'ai eu un contact en particulier pour le syndicalisme clandestin et ce contact parisien que l'on avait, c'était... son pseudonyme c'était Friquet, Robert Hernio qui doit être décédé maintenant, puisqu'il habitait en Bretagne, et donc, périodiquement, il venait nous voir et avec un cheminot du dépôt de Tourcoing, Charles Belin, nous étions les deux contacts de ce dirigeant national qui..., qu'on rencontrait tous les 15 jours-trois 
semaines, enfin périodiquement, et à partir de ça, il donnait des instructions, « essayez de faire ceci, essayez de faire cela »; et il faut dire qu'au dépôt de Tourcoing nous sommes arrivés à fomenter une grève, en août 43, ce qui était assez rare, et on a pu utiliser le prétexte suivant: quatre de nos camarades de travail étaient mutés par la direction dans un autre établissement sous le prétexte « insuffisamment productifs ». Alors on a réussi à utiliser ce prétexte pour faire arrêter le travail pendant quelques heures, un après-midi en août 43 , ce qui était très dangereux bien entendu, hein, et d'autre part, eh bien évidemment, on diffusait toute la presse Front national...

- Tu te rappelles les titres?

- Eh bien, il y avait le journal du Front national, Franc tireur, il y avait tous les journaux édités par le Front national et très souvent il arrivait qu'on n'ait pas suffisamment de matériel et avec Charles Belin, on se réunissait un soir et puis on discutait, on peut parler de ceci de cela, je rédigeais un texte et avec la pâte à polycopier on faisait une série d'exemplaires, mais avec une matrice de pâte à polycopier, on faisait 20 exemplaires, il fallait donc que je recommence $\mathrm{X}$ matrices pour pouvoir refaire 100 ou 150 exemplaires, et on y passait toute la nuit. On a fait ça tous les deux au moins trois ou quatre fois quand même, hein.

- Et comment se faisait la diffusion?

- Et c'est nous qui faisions la diffusion, évidemment comme tous les journaux clandestins, à l'abri des regards indiscrets...

- Sur les lieux de travail?

- Sur les lieux de travail, les armoires vestiaires essentiellement.

- Alors, petit à petit il y a une prise de risques progressive qui se fait, qui conduit à chercher à nuire à loccupant, de quelle facon?

- Tout d'abord, eh bien, j'ai commencé à imaginer, et puis le réseau Sylvestre avait commencé à naitre, et puis j’ai fait la connaissance, enfin, appris que Paul Cousaert...

- Il faut expliquer pour les plus jeunes que Paul Cousaert, c'est un réseau francobritannique.

- Oui, c'était un réseau franco-britannique qui était monté par un capitaine..., officier anglais, le capitaine Michel, son nom : Michael Trotobas, qui était à l'un des réseaux du SOE dirigé sur l'ensemble de la France par le colonel Buckmaster. Et, donc, Paul Cousaert qui

- La Coupole / Georges Delepaut. Citation autorisée dans un cadre scientifique à 
était entré dans ce réseau, ben on discutait tous les deux ce qu'on peut faire, et puis on a pensé, en particulier, à essayer de provoquer des chauffages de boittes d'essieux.

- Ceci aussi est important, comme quoi tu es cheminot tu connais bien la chose, comment on peut arriver à paralyser une machine discrìtement?

- Alors, les locomotives à vapeur et les wagons à l'époque, les essieux tournaient dans des boites avec des coussinets, des coussinets qui étaient garnis d'un métal appelé antifriction, mais qui évidemment étaient graissés avec de l'huile. Il y avait donc une réserve d'huile et, cette huile devait être pure, s'il y avait des impuretés dedans, ça arrivait à provoquer ce qu'on appelait un chauffage, c'est-à-dire que des impuretés qui venaient, ça faisait chauffer l'essieu, ça pouvait aller très loin puisque ça pouvait faire rougir et même une rupture d'essieu. Alors, notre technique était la suivante : comme il y avait à notre disposition des poudres abrasives pour faire le rodage des clapets, des robinets qui sont nombreux sur une locomotive à vapeur, eh bien on utilisait un petit peu de cette poudre qu'on mettait en petite quantité, dans les petits bacs de réserve d'huile qui alimentaient chacune des boites d'essieux. Alors, bien sûr, un chauffage de boîte d'essieux sur une locomotive, ça peut..., ça paraît normal, bien que le dépôt de Tourcoing qui était un dépôt, logiquement, normalement, [qui] pouvait avoir une boite chaude par mois et encore, on en est arrivé, à un moment, à en avoir cinq et six par semaine. Des enquêtes ont été faites, rien n'a été découvert, ce qui fait qu'il fallait, pour nous, surtout éviter qu'il y en ait deux sur une même locomotive. Ce qui fait qu'avec Paul Cousaert, on disait, « Paul, attention, $j$ 'ai fait une boîte chaude à telle locomotive », il savait qu'il devait pas y aller. On s'arrangeait comme ça. Et puis on a même imaginé tous les deux de faire des montages d'embiellage de distribution de vapeur, sur des locomotives, à l'occasion d'une modification qui se faisait, un montage tel, ben, que il y avait un axe qui partait, donc il y avait une avarie qui produisait en ligne. Il est évident que ce soit une boite chaude ou n'importe quelle avarie, sur une locomotive, ça se produit toujours lorsque cette loco est en tête d'un train, en train de remorquer à grande vitesse. Ce qui fait que sur la voie, en pleine ligne, le train se trouve bloqué, ce qui est derrière l'est également, et puis, bon, il faut venir sur place, enlever la machine, en amener une autre, emmener celle-là, faire la réparation et il y a donc un retard important qui se produit pour ce train et pour ceux qui sont derrière. De même qu'un déraillement se produit, là c'est pareil, fallait dépanner, fallait remettre sur les voies. La voie contiguë était bloquée pendant un 
certain temps, mais cette voie évidemment était assez rapidement dégagée pour laisser passer les trains quand même, qui passaient au pas, mais qui passaient. Par contre, le capitaine Michel avait envisagé, avant d'être abattu le 27 novembre 1943 à Lille comme on le sait, eh bien il avait envisagé de provoquer des avaries sur les locomotives pour les rendre indisponibles pendant un certain temps. Ce qui fait que, ben, un jour, en décembre 43, Paul Cousaert, un après-midi, le 20 décembre, est venu me dire, "Eh bien, écoute, on va faire une opération ». C'était Arthur Malfait, l'un des adjoints du capitaine Michel qui par conséquent avait pris l'initiative de réaliser une de ces opérations imaginées par le capitaine Michel, qui malheureusement, était disparu depuis le 27 novembre.

- Il faudrait peut-être dire que le capitaine Michel était un professionnel de l'action qui donc avait ciblé des objectifs...

- Oui, oui, il avait ciblé des objectifs économiques, les chemins de fer bien sûr parce que c'était très important pour les Allemands qui, manquant de carburant, avaient intérêt à faire transporter le maximum de leur matériel, de leurs hommes, de leur ravitaillement par voie ferrée, hein, et économiser le carburant. Et si on gênait les transports, c'était toujours intéressant, de même que les voies fluviales ont été également l'objet d'attaques, de même que les lignes téléphoniques pour les communications. Donc, pour cette attaque-là, le 20 décembre après-midi, Paul Cousaert est venu me voir, enfin l'après-midi, et m'a dit : «Tu veux venir avec nous, on va faire une opération ce soir, cette nuit, là, contre le dépôt, on fout des locos en l'air... », « D’accord ! », alors rendez-vous est pris pour $23 \mathrm{~h}$. Nous nous retrouvons à $23 \mathrm{~h}$ au dépôt, ensemble, et là les charges qui avaient été préparées l'aprèsmidi dans une maison du quartier, avaient été amenées, par Paul Cousaert d'ailleurs, au dépôt et à $23 \mathrm{~h}$ nous nous réunissons dans un bâtiment qui était la lampisterie, nous étions cinq. Il y avait donc Arthur Malfait, qui n'était pas cheminot lui, il y avait Gabriel Royer qui était cheminot et trois jeunes du dépôt, Jean Debruyne, Paul Cousaert et moi. Et donc, nous choisissons, Arthur Malfait, Paul Cousaert et moi, les locomotives, et donc nous prenons les charges, prévues pour ça, c'est qu'il y avait des retardements de $2 \mathrm{~h}$.

- C'était du matériel anglais?

- Matériel anglais, qui provenait des parachutages dans le Pas-de-Calais en particulier. Et les deux autres devaient pénétrer dans l'atelier par la fenêtre du fond dont j'avais tourné l'espagnolette avant de partir, en fin de service, de façon qu'on n'ait qu'à la pousser. Pour les 
machines-outils, les retards prévus étaient d'une demi-heure, retard que l'on connaissait par la couleur de la goupille de sécurité qui était sur le crayon à temps. Et nous partons, Paul, Arthur et moi, d'abord on met des charges sur deux locomotives à la force roue [?] où on réparait les chauffages de boites d'essieux, ça faisait la queue là, deux locomotives prêtes à partir, on leur dit deux mots, et ensuite neuf locos dans la rotonde. Malgré la présence de sentinelles allemandes dans la rotonde, du poste de garde allemand, des cheminots allemands qui étaient toujours là présents évidemment $24 \mathrm{~h}$ sur 24 et qui étaient armés, et de cinq Français, payés pour surveiller, mais comme rien ne se passait jamais, les gars étaient, fin décembre, en train de se chauffer autour d'un brasero. Nous sortons, les trois de la rotonde, on retrouve les deux autres qui n'avaient pas pu pénétrer dans l'atelier, quelqu'un mal intentionné - «Tiens, on a oublié de fermer la fenêtre ! » - et il l'avait fermée, et par conséquent les copains voulaient abandonner et je dis: «Non, on va y aller», je vais chercher un morceau de ferraille, je vais casser la fenêtre, et puis après on a attendu quelques instants pour être certains que le bruit n'a pas été perçu, je suis entré par la fenêtre, j'ai ouvert la porte et là tout le monde est entré et on a posé les charges sur les machines-outils. Neuf machines-outils, deux vérins de base, j’avais quatre vérins, des grands vérins qui permettaient de lever complètement une locomotive pour faire de grosses réparations. Neuf machines-outils, sur ces neuf machines-outils, six vont aller à la mitraille, les trois autres nécessitaient des réparations longues, mais pas de remplacement par des machines-outils neuves parce que tout ce qui était construction neuve partait en Allemagne pour remplacer ce qui était démoli par les bombardements. Et onze locos, trois, la première remise en service fin février, progressivement remises en service, le $1^{\text {er }}$ mai 1944, quatre encore pas réparées. L'atelier, bloqué, et les machines-outils qu'il a fallu trouver à droite et à gauche, l'atelier a été inopérant pour l'entretien jusqu'en avril 1944. Ce qui fait que des locos manquaient à l'appel pour remorquer des trains, donc le but visé par le capitaine Michel était bien atteint, et, en plus, l'atelier inopérant obligeait les locos qui devaient passer en révision ou en entretien, des réparations, a être groupées en convoi, acheminées à Lille-Délivrance où là elles attendaient leur tour parce que Lille avait les siennes également, et au lieu d'être immobilisées, si Tourcoing avait été opérationnel, leur immobilisation durait quelquefois jusqu'à une semaine, donc du temps supplémentaire où elles manquaient à l'appel pour remorquer des trains. 
- Alors, bien évidemment, les Allemands vont réagir à cette opération. Peux-tu nous dire en quelle circonstance?

- Alors, les Allemands, évidemment, bon, c'était... ça apparaissait comme le nez au milieu de la figure qu'il y avait des spécialistes dans l'affaire. Ils avaient donc arrêté tous les gars qui avaient travaillé pendant la nuit du 20 au 21 et qui avaient travaillé jusque, en soirée, jusqu’à $22 \mathrm{~h}$. Et parmi eux, un jeune, tourneur, son père comme les membres des familles des autres gars, portait à manger, le matin, le midi et soir, là où ils étaient, et deux jours après, un midi, les Allemands ont conservé ce gars avec eux. Et ce gars devait connaitre un peu les gens, pensait que untel, untel devait appartenir... il est resté avec les Allemands le midi et comme par hasard, l'après-midi même, les Allemands venaient au dépôt et demandaient Paul Cousaert. Ils étaient deux, le père et le fils, qui se prénommaient de la même manière, donc, c'est le père qui s'est présenté, ils sont repartis avec lui. À leur arrivée là-bas, l'intéressé à dû dire : «Non, c’est pas lui, c'est le fils. » Ils sont revenus, on avait fini la journée, par conséquent chacun était reparti, mais Paul savait bien que ce n'est pas à son père qu'on en voulait mais à lui. Il est donc passé chez sa mère pour l'aviser, il était chez lui, en train de préparer des vêtements, pour partir dans la clandestinité, mais évidemment pendant ce temps-là, les autres étaient passés au dépôt, avaient l'adresse, et il était encore chez lui, il n'était pas encore parti lorsqu'ils sont arrivés. Il a donc été arrêté, on sait qu'il a été torturé, il a subi le casque avec les vis pénétrant dans la boîte crânienne, et il a été déporté en Allemagne et on sait par un de ses camarade du camp comment il est décédé au cours du transfert d'un camp à un autre avec l'avance des Russes. À trois, un soir, en janvier 1945, ils ont tenté l'évasion, deux ont été repris, dont lui, ils ont bien sûr été abattus sur place, et le troisième a réussi à rester caché, c'est comme ça qu'on le sait. Et, par conséquent, il a été libéré par les Russes deux jours après. Il est revenu avec les Russes sur place pensant enterrer ses copains, mais les Russes n'ont pas laissé faire parce que souvent les SS minaient les cadavres pour les faire péter quant on y touchait.

\section{- Quel âge avait Paul Cousaert lorsqu'il a été arrêté?}

- Paul Cousaert est de 21, de juillet 1921.

- Donc, l'arrestation de Paul Cousaert, c'est un signal?

- C'est un signal, automatiquement, un gars arrêté, l'esprit de sécurité nous a obligés, les quatre autres, à partir dans la clandestinité. 
- Alors, c'est pour toi, c'est un changement de vie?

- Un changement de vie commence, me voilà clandestin, et bien sûr, comme j'étais entré ... à mon entrée au Front national, j’avais adhéré aux Francs-tireurs partisans, qui étaient la branche militaire du Front national, je suis parti dans les FTP, j'étais donc devenu un permanent des FTP hébergé d'abord à Roncq puis à différents endroits comme chacun sait.

- Avant qu'on aborde ta vie clandestine, Georges, j’aimerais que tu expliques un peu pour les gens d'aujourd'hui qui connaissent très mal les chemins de fer de l'époque, et qui ont souvent une vision un peu mythologique, on voit toujours le déraillement comme étant l'action symbolique de la résistance française entre 1940 et 1944. Est-ce qu'il n'y a qu'une seule méthode pour faire dérailler un train?

- Oh non, il y avait évidemment plusieurs façons d'opérer, il y avait une façon manuelle, disons, qui, au moyen des clés utilisées normalement pour faire l'entretien et la mise en place des voies, hein, de déboulonner des bouts de rail, avec, au droit d'une éclisse, à la jonction entre deux rails, et parce que à l'époque les rails n'étaient pas continus, c'étaient des longueurs mises bout à bout, et enlever les éclisses, et puis le rail tordu, pour faire partir sur le côté de la voie, et donc déraillement. Autre moyen, au moyen d'explosifs. Avec des charges, placées de part et d'autre des rails, avec un contacteur, qui est placé un peu en avant, lorsque la locomotive..., le premier essieu va faire claquer par conséquent les charges, et puis, un morceau de rail qui manque, et par conséquent, il y a déraillement également. Alors, après, évidemment, il y avait le choix de l'endroit pour pouvoir le faire, quelquefois le choix n'était pas très judicieux, mais souvent il était bon quand même.

- Les courbes?

- Les courbes, les talus, ça dégringolait, en particulier quand il s'agissait d'un convoi militaire avec du matériel, ça faisait assez bien de dégâts. Quelquefois en pleine ville, c'était à éviter, malheureusement, ça a été le cas à Ascq. On sait ce qui en a suivi.

- On en vient maintenant à la période de ta vie clandestine. Quel est le quotidien d'un clandestin, qu'est-ce que ça signifie être clandestin chaque jour?

- Être clandestin, c'est déjà évidemment, avoir une autre identité, à laquelle il faut s'adapter, connaitre ce nom, et puis essayer de choisir quelque chose, qui, notamment au point de vue prénom et appellation habituelle, ressemble un peu à... alors, moi, c'était 
Georges, on m’appelait Jo, donc j'ai choisi comme prénom Joseph et comme j'avais un cousin qui s'appelait André, on l'appelait l'André, j'ai choisi le nom de Landré, j'avais déjà un petit peu ces trucs-là, un petit peu habitué à ça. Parce que évidemment, si on m’appelait Jo et qu'il y avait des gens là et qu'on vérifiait l'identité, et que c'était tout à fait un autre prénom, ça pouvait être bizarre. Ça c'est un premier point. Alors, après, évidemment, là, c'est... sachant que bien sûr on risque l'arrestation, que peut-être bien le copain qui a été arrêté, sous la torture, peut-être va parler, il faut savoir qu'on peut être recherché, et par conséquent, essayer d'éviter, et être toujours aux aguets, on est dehors, et même à l'intérieur quand on est dans une maison, on n'est jamais à l'abri, il peut toujours y avoir une descente et être arrêté. Donc c'est constamment être aux aguets pour essayer d'échapper, et puis, toujours être en train d'observer, s'il va y avoir une rafle qui se prépare, essayer d'éviter d'être dedans, toujours essayer d'éviter d'être dans la grande foule et d'essayer... Les cinémas, plus question, les sorties, plus question, c'est évidemment faire son boulot, aller à ses rendez-vous et faire ce qu'on a à faire simplement.

\section{- Il faut être planqué aussi ?}

- Être planqué, mais la planque, les gens qui nous hébergent, qui risquent gros, obligatoirement on est obligé de changer périodiquement, tous les quinze jours, trois semaines, il fallait changer et puis il fallait retrouver d'autres Français patriotes qui acceptaient de nous héberger parce c'était très dangereux pour eux.

- Tu peux faire quelques portraits de ces gens qui t’ont hébergé?

- Des gens qui m’ont hébergé par exemple, je me souviens de personnes, la dame vit encore, le monsieur a malheureusement été arrêté d'ailleurs après, des gens qui habitaient rue de Verdun à Tourcoing, qui étaient des amis d'une de mes sœurs, ma sœur qui était plus âgée que moi, et qui ont accepté de m’héberger trois semaines à peu près, après mon départ, puisque au début j'étais à Roncq, après je suis revenu là, je suis reparti à Roncq, je suis revenu à Tourcoing, enfin, différents endroits. Et ces gens m’ont d'abord hébergé, ensuite je leur ai demandé s'ils pouvaient me mettre à l'abri du matériel, et puis après je leur demandé s'ils pouvaient accueillir des réunions nocturnes d'état-major, et malheureusement comme il y a eu un gars qui a réussi à s'infiltrer dans les rangs et arriver à ce niveau-là, une arrestation importante s'est faite en avril 1944, à Tourcoing, rue Dugay-Trouin et, là, le gars qui avait dénoncé connaissait également ces gens-là et 
puis le monsieur a été arrêté, il a été déporté, il a fait des prisons successives, il a fait dix-sept prisons et il n'a été libéré, il n'est rentré qu'en septembre 1945.

- Donc, tu te déplaces, tu changes de plan, pour l'argent, pour la nourriture...?

- Alors, pour l'argent, le mouvement FTPF disposait d'argent qui, pour la plupart du temps, était de l'argent qu'on allait chercher, les payes de grosses entreprises, des choses comme ça. Pour ma part, j’ai eu à participer une fois au moins à aller prendre la recette d'un gros chevillard qui sortait de l'abattoir de Lille. Par contre, les tickets de ravitaillement, on allait se servir dans les mairies, on faisait une attaque. En règle générale, avec des complicités au départ quand même, hein, à l'intérieur. J'ai participé à une attaque à Roncq par exemple, donc on avait les tickets de ravitaillement, on en avait plus, on nous en donnait plusieurs jeux, de façon à pouvoir en donner davantage aux gens qui nous hébergeaient, en remerciement. Et l'argent qu'on avait, évidemment, c'était pour vivre, donc on leur payait également la nourriture. Et puis le reste c'était pour vivre, nos déplacements, nos transports en commun, il fallait bien vivre quand même, mais pas de distraction évidemment.

- Quelles sont les activités que tu es amené à entreprendre, en tant que clandestin, dans les cinq à six premiers mois de 1944 ?

- Alors, dès que je suis clandestin je deviens l'adjoint de Joseph Catry qui était le responsable FTP qui m'avait recruté, qui était le technicien du secteur, et qui malheureusement était malade, et par conséquent, je suis immédiatement devenu son adjoint et lorsqu'il n'a plus été capable, je suis devenu le technicien pour la région, donc, le matériel qu'on pouvait avoir, si on avait besoin de préparation de charges, pour un endroit ou pour un autre, c'était moi qui devait préparer tout ça. À l'occasion, j'ai préparé, avec des boîtes de conserve et des bouts de bois, des contacts, des pédales pour faire le contact, pour provoquer des déraillements par exemple, des choses comme ça.

- Est-ce que tu peux préciser l'organisation interne des FTP ? Y a-t-il une répartition des tâches présentée?

- Alors, la répartition des tâches, un détail c'était des trinômes en fait. Alors, il y a un gars qui est le chef militaire, qui règle l'organisation, il $\mathrm{y}$ a un gars qui est le financier, tout ce qui est tickets de ravitaillement et finances, et puis il y a le troisième qui est l'aspect plus politique de l'affaire, l'orientation de l'affaire. Et les trois ont évidemment des contacts avec leur correspondant au niveau régional, par secteur. Et 
entre eux, en liaison avec les contact directs du..., moi, mon chef direct c'est Marcel Verfaillie qui était arrêté à Tourcoing là, commandant Guy, en parallèle avec ça, il y a les agents de liaison, qui doublent les contacts de façon à permettre de renouer ceux-ci dans le cas où on loupe un rendez-vous. Parce qu'évidemment, une alerte obligeait... les transports en communs arrêtés, obligeait d'être à l'abri, et puis les rendez-vous étaient loupés. Donc, le contact pouvait se renouer au moyen des agents de liaison.

- Quelles sont les actions auxquelles tu participes dans les premiers mois de l'année 1944, dans le cadre de ta vie clandestine?

- Alors, pour moi, essentiellement c'est en tant que technicien, mais lorsque j’ai été appelé à aller chercher, dans le Pas-de-Calais, à Nœuxles-Mines d'ailleurs, du matériel qui nous parvenait par parachutage, ça doit être fin janvier, début février, ce matériel, là évidemment on l'a ramené à Tourcoing, d'ailleurs avec un épisode tout à fait particulier. Nous avions été demandés, deux gars, qui étions dans l'illégalité, d'aller à Nœux-les-Mines pour chercher du matériel. Alors, évidemment, comme d'habitude il y avait peu de choses, on était parti avec des valises. Arrivés là-bas, Pfff! Oh, les valises! Heureusement il y a un camion, un camion benne, on avait 25 mitraillettes, un fusil mitrailleur, un fusil antitank, des explosifs, enfin... des mines anti-personnels sous forme de crottin de cheval, enfin tout ce qui existait... et nous étions cinq, nous deux et trois gars qui étaient avec nous. Et le gars qui pilotait le véhicule, qui parlait un peu l'allemand, mais ne connaissait pas la région, on est partis, le soir commençait à arriver, et le gars, ne connaissant pas la région, s'est arrêté au niveau d'une sentinelle allemande, à qui il a demandé son chemin. Et l'autre, évidemment, lui a indiqué : «Pour partir, c'est par là. » Le gars n'était pas curieux, il a pas cherché à savoir ce qu'il y avait là-dedans, parce qu'il serait mort, mais nous aussi, parce que, évidemment, on serait pas allé loin. Et nous sommes arrivés à Tourcoing, chez les beaux-parents de Joseph Catry qui tenaient un commerce de boissons, eaux, sodas, bières..., enfin toutes les boissons, et donc on a mis le camion à l'abri là, pour le reste de la nuit. Et le lendemain on est allé transporter ça à Roncq, où on a planqué tout ça, et distribué après. Et ce matériel a donc servi, ça doit être en février, peu de temps après, moins de quinze jours après, et je suis allé avec un groupe de Roncq à qui j'avais demandé de m'accompagner, dire deux mots à un pylône de ligne à haute tension qui joignait Comines à Wasquehal sur le territoire de Linselles. Evidemment pylône choisi à un changement de direction de façon 
à ce qu'il se casse la figure parce que si on prend un pylône en ligne droite, les pieds sont coupés, mais il reste debout maintenu par les câbles. Tandis que là, ça fait deux feux d'artifice, l'explosion d'abord, et puis quelques secondes après, quand tout se casse, les flashes électriques et puis après toute la région est dans le noir immédiatement. Alors là, c'est la joie, comme à Tourcoing, la joie intense d'avoir réussi son opération. Et puis après, d'autres opérations encore, auxquelles j’ai participé et, lorsque j'ai été arrêté à Saint-Quentin, parce que suite à l'arrestation en avril à Tourcoing, je savais qu'il y avait une réunion à Saint-Quentin, pour une répartition de matériel, javais entendu parler: "Saint-Quentin, $17 \mathrm{~h}$, gare » et je m'étais imaginé $17 \mathrm{~h}$ à la gare de Saint-Quentin. Je me débrouille pour y être au jour dit, à $17 \mathrm{~h}$ à la gare de Saint-Quentin, mais personne, évidemment, je tourne, et comme il y avait eu un commissaire de police collabo qui avait été abattu quinze jours auparavant, les flics français étaient sur les dents, et puis repéré et vérification d'identité, évidemment identité fausse, mais j’avais pas d'arme, rien du tout, j’avais une valise avec des bleus, parce que, le prétexte, j’allais chercher du travail, j'étais un réfractaire, j’avais l'âge des STO. Et par conséquent vérification d'identité, j’ai refusé de dévoiler mon identité au départ, parce qu'il fallait laisser le temps aux gens qui nous hébergeaient ou faire évacuer les copains, évacuer tout ce qui était chez eux, on ne savait jamais, et donc au bout de quelques jours, véritable identité, et puis, bon, à Tourcoing les flics qui ont répondu, ont dit qu'il n'y avait rien contre moi, alors qu'on savait que les Allemands, en principe, devaient plutôt chercher à nous avoir.

\section{- En fait, la police française commençait un peu à changer de doctrine?}

- Oui, on était à fin avril, ça commençait évidemment à bouger un petit peu, et donc, là, le jour où j’ai été arrêté, le soir même j’ai été enchainné là à un radiateur. Un coup de téléphone, on demande une patrouille, avenue de la Chaussée-Romaine, à Saint-Quentin, un lieutenant de GMR interpellait un suspect. Et puis ils s'en vont, ils reviennent, et qui je vois arriver? Un interrégional que je connaissais bien, Gaby.

\section{- Un interrégional FTP?}

- Un interrégional FTP, que je connaissais, qui était un de ceux qui devait être à cette réunion et c'est là que j'ai compris que les $17 \mathrm{~h}$ c'était à la gare de Lille pour aller à Saint-Quentin, et non pas $17 \mathrm{~h}$ à SaintQuentin. Alors, ils étaient en train de chercher la maison dans laquelle il devait se rendre, et cet officier GMR qui habitait à proximité a eu peur, a sorti son arme, et évidemment a réussi à interpeller, mais lui, 
malheureusement, il a été pris avec des papiers sur lui. Donc là, on a passé quelques jours dans les cellules du commissariat de SaintQuentin, ensemble, on a donc pu parler, et je lui ai expliqué ce que j'imaginais comme processus de défense, et il m'a dit : «Oui, bon, on peut essayer. » Et puis au bout d'une semaine il a été transféré à Paris, et je ne sais pas ce qu'il est devenu d'ailleurs, et moi, quand j'ai dévoilé mon identité, j'ai expliqué que j'étais un simple réfractaire au STO et que chez moi était venue une dame bien mise, manteau de fourrure, décrivant quelqu'un qui semblait issu de la haute bourgeoisie, qui m'avait proposé une carte d'identité, des tickets de ravitaillement, tout pour m'aider à ne pas partir au STO. Alors... Tourcoing a répondu certainement plutôt favorablement, et au bout de quinze jours, condamné à quinze jours de prison avec sursis, c'était déjà fait, et le soir même rentré à Tourcoing, pas chez moi bien sûr, mais deux jours après, la police de Saint-Quentin était chez mes parents, savait qui jeétais, je ne les avais pas attendus évidemment. Alors comment ils l'ont su, je n'en sais rien. Est-ce que quelqu'un, Tourcoing a donné un renseignement quand même, ou, est-ce que le gars qui avait été arrêté, me sachant arrêté, pour parler de quelqu'un a parlé de moi et de personne d'autre, je n'en sais rien, toujours est-il que ça s'est passé comme ça. Et là, bon, ben, j’ai passé un moment à Tourcoing, ensuite je suis arrivé à Roubaix où j’ai été nommé commandant de compagnie FTP qui commençait à se monter sur Roubaix, la Compagnie Domisse.

- Alors avant d'en venir aux événements qui vont suivre le débarquement du 6 juin, il s'est produit au mois d'auril 1944 dans la région lilloise deux événements très importants qui ne powvaient que toucher un cheminot comme toi. Le premier événement c'est, bien sûr, le massacre d'Ascq, qui est la conséquence d'un sabotage réalisé par un petit groupe de la Voix du Nord. Comment est-ce que la nouvelle a été perçue par les cheminots, de ce massacre, quels étaient les enjeux qui ont étéperçus?

- Ben, quand ça s'est produit, moi je n'étais plus parmi les cheminots, mais par contre, évidemment on en a entendu parler, on a su, bien sûr, que c'est ça, puisque on sait que les journalistes qui ne pouvaient expliquer ce qui c'était passé, avaient trouvé l'astuce de publier le même jour, les 86 noms des victimes d'Ascq, et par conséquent tout le monde a compris qu'il y avait eu quelque chose qui s'était passé. Par contre, ce qu'on savait bien, c'était surtout les bombardements.

- Avant de parler des bombardements, est-ce que il n'y a pas eu des discussions au sein des groupes de résistance pour l'attitude à adopter en cas de débarquement justement? 
- Ben, non, évidemment pour nous les consignes étaient des attaques en particulier contre les chemins de fer, des déraillements étaient provoqués, il fallait essayer d'éviter justement les zones urbaines. Il fallait essayer de faire ça en campagne, de façon à ce qu'il y ait pour eux un temps de réaction avant d'arriver à quelque chose. Par contre, les bombardements, alors là il y a eu Lille-Délivrance en avril à Pâques 1944 où là, on sait que c'était les Américains qui avaient bombardé avec leur technique du tapis qui descendait et on sait le nombre de morts qu'il y a eu. Par contre, Tourcoing, la suite de notre opération a été à l'abri de bombardements pendant plusieurs mois. Et le premier bombardement qu'il y a eu sur Tourcoing, ça été le 13 mai 1944, où là, on sait que c'est le groupe Lorraine qui a fait le bombardement, et chaque avion a visé à tour de rôle, d'ailleurs j'ai chez moi une reproduction de la photo prise par le dernier avion, on voit tous les impacts bien groupés, quelques-uns en dehors de l'enceinte SNCF, mais tout bien groupé, et il n'y a eu, pour un bombardement de cette importance, quand même que, quand même huit morts, ce qui était peu, alors que nous, nous n'avons pas eu des morts dans la population civile, par contre, malheureusement, au dépôt de Tourcoing, on a eu un gars, un mort, mais accidentel. C'est un visiteur de machine qui était de service, et comme les Allemands n'osaient pas venir voir si les locomotives pouvaient partir ou pas, ils se sont mis à hurler tellement sur lui qu'il a eu plus peur des vociférations des Allemands que des risques d'explosions qui avaient commencé, et ils sont allés à deux, et au moment où ils passaient dans la rotonde, ils étaient deux, une charge a explosé, puis le gars a pris un morceau de tôle dans la cuisse et il a eu l'artère fémorale sectionnée. Ce qui fait qu'il a été transporté vers 1 heure et quart le 21 décembre au matin, il est mort quelques heures après, à $6 \mathrm{~h}$ du matin à l'hôpital de Roubaix. Mort accidentelle.

- Pour en revenir aux bombardements du printemps 1944, on s'apercoit qu'il y a toute une série d'opérations qui sont montées par les Anglais et les Américains, contre Lille, contre Somain...

- Oui, tous les centres SNCF importants sont visés, les dépôts, les triages, tout, tout est visé, semblant indiquer que bon, ben, il faut détruire tous les moyens de transport parce que, ben, si un débarquement qui va se faire, il faut que tous ces moyens-là soient... On sait rien.

\section{- Et il commence où le débarquement?}

- On sait rien, à l'époque on ne sait rien par contre c'est dans la deuxième quinzaine de mai, je suis envoyé dans le sud des Ardennes, avec un 
gars de Douai, un gars de mon âge, nous sommes envoyés dans la... à un rendez-vous avec un interrégional, Nicolas, qui nous donne rendez-vous à une intersection de routes. On s'en va le jour $\mathrm{J}$ et puis arrivés là, son agent de liaison, Claire, vient, nous dit : « Nicolas n’est pas là, il ne viendra que demain, il a été retenu par une réunion. » Le lendemain, on est là tous les trois à l'attendre, il arrive, et il nous explique : «Écoutez les gars, retour maison, mission supprimée », et il nous a expliqué ce qui nous attendait normalement. On devait avec lui, préparer des positions de repli pour nos groupes qui auraient reculé en même temps que les Allemands, suite au débarquement qui était prévu. Mais ça ne vous disait pas où le débarquement devait se faire.

\section{- Mais la population pensait que cétait dans le Nord-Pas-de-Calais à l'époque?}

- Ben, étant donné que c'était l'endroit le plus court et puis l'importance des bombardements faits dans notre région ont fait croire, et d'ailleurs on sait qu'il y a eu une opération d'intoxication anglaise, l'opération "Fortitude » qui a fait croire aux Allemands que le véritable débarquement serait sur les côtes de la mer du Nord. Ce qui fait que le 6 juin, il y a eu la Normandie, ils ont cru à une opération de diversion.

- Alors, le 6 juin, ça va bien sur relancer l'activité des groupes locaux de résistance?

- Oui, évidement, il faut reprendre, et puis surtout, les transports à essayer de gêner. Toujours, l'objectif principal, c'est ça, gêner les transports, et c'est là qu'il y avait déjà eu des attaques contre les voies fluviales, en particulier le réseau Sylvestre qui a attaqué des écluses notamment, et puis on sait qu'à la libération par exemple, le canal de Roubaix était à sec. Et tout ça était destiné à empêcher le transport, en particulier des matériaux, du ciment, pour les montées, les rampes de lancement qui étaient entrain de s'installer pour les V1 et les V2. Tout ça aussi, ça été un objectif en plus du renseignement qui était toujours aussi demandé. Essayer, chaque fois qu'un renseignement pouvait être recueilli, des unités qui arrivaient, repérer..., ces renseignements-là étaient toujours fournis et remontaient bien sûr pour arriver aux Britanniques.

- Alors pendant cette période qui va du 6 juin au début septembre 44, jimagine que les Allemands vont être encore plus nerveux que d'habitude. Comment ça se traduit?

- Oui. Ça se traduit par des arrestations qui arrivent de plus en plus nombreuses, mais alors moi j'ai eu l'impression, durant le mois d'août 
1944 en particulier, il y avait plus d'arrestations, mais on se sentait mal à l'aise, comment ça se fait, on est épié, on s'attendait à ce qu'il y ait des rafles monstres, heureusement, la libération est arrivée.

- Ily a en quelques rafles ponctuelles, je crois?

- Oui, mais pour nous, dans nos rangs, nous les gens qui étions dans la clandestinité, on était assez inquiets quand même de se rendre compte qu'il n'y avait pas d'arrestations, alors qu'il y en avait de plus en plus, c'était de plus en plus fréquent et puis, là, y en avait pas. Alors là on se disait ça y est, ils sont en train d'essayer d'en repérer un maximum pour faire une rafle monstre, quelque chose de monumental, et heureusement, le $1^{\text {er }}$ septembre est arrivé. 